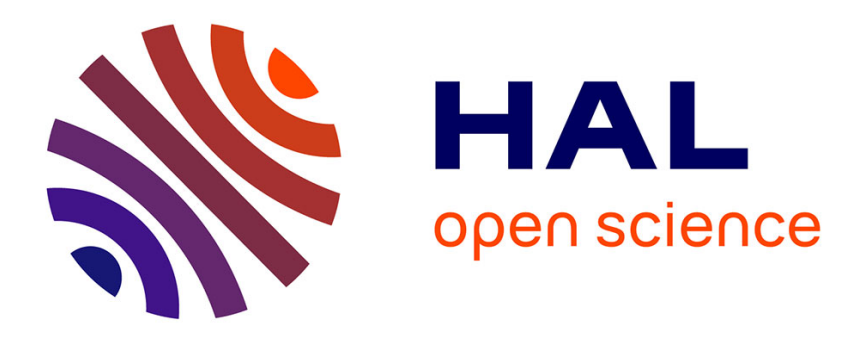

\title{
Tombeau photographique pour les mutins assassinés d'Attica
}

Philippe Artières

\section{To cite this version:}

Philippe Artières. Tombeau photographique pour les mutins assassinés d'Attica. Sociétés \& Représentations, 2020. hal-03090483

\section{HAL Id: hal-03090483 \\ https://hal.science/hal-03090483}

Submitted on 29 Dec 2020

HAL is a multi-disciplinary open access archive for the deposit and dissemination of scientific research documents, whether they are published or not. The documents may come from teaching and research institutions in France or abroad, or from public or private research centers.
L'archive ouverte pluridisciplinaire HAL, est destinée au dépôt et à la diffusion de documents scientifiques de niveau recherche, publiés ou non, émanant des établissements d'enseignement et de recherche français ou étrangers, des laboratoires publics ou privés. 


\section{Tombeau photographique pour les mutins assassinés d'Attica}

Philippe Artières

À Elisabeth Fink in memoriam

Dans la galerie parisienne Marian Goodman, je découvre une œuvre de l'artiste contemporain Steve McQueen intulée $A s h e s^{1}$ - c'est un dispositif très simple : sur 1'écran, on voit un jeune homme métis dansant sur la proue d'une barque au milieu de la mer des Caraïbes, il est très beau, plein de vie, plein d'énergie; au dos de l'écran, une seconde vidéo montre un vieil homme, dans un cimetière de la Barbade, en train de construire minutieusement et avec soin une tombe sur laquelle il finit par graver le nom du jeune homme vu sur l'autre face. On apprend par un texte que le jeune homme était un dealer et qu'il a été tué lors d'un règlement sa redoutable efficacité. Ériger un monument pour les morts est la tentation de l'historien quand il a soudain en héritage leur dernière image. Mais plutôt que de figer ces morts en gloire comme l'artiste, de réaliser un monument funéraire, le rôle de l'historien est de rédiger avec leurs images un «tombeau » qui n'est pas élection individuelle — l'historien ne dresse pas un Panthéon - mais qui raconte une histoire collective. Récit d'une tentative.

La scène se passe à Brooklyn un après-midi de janvier 2015 ; j'avais quitté Paris quelques heures plus tôt, le lendemain des attentats de Charlie Hebdo et du magasin Hyper Casher. Les auteurs des attaques étaient encore en cavale. Les images de la fuite des terroristes tuant un policier à bout portant sur le boulevard tournaient en boucle sur les écrans de l'aéroport, la liste des victimes ne cessait de s'allonger.

Je suis à New York, car j'y mène une enquête sur une mutinerie de prisonniers du 9 au 13 septembre 1971 à Attica, l'un des plus grands centres pénitentiaires de l'État de New York. Ce soulèvement d'un millier de détenus, majoritairement afro-américains, portoricains et hispaniques avait fait doublement événement: par sa médiatisation immédiate (les mutins ayant obtenu dès les premières heures l'entrée d'une équipe de télévision), elle est la première mutinerie filmée en direct; par sa répression violente, elle est considérée, en 1971, comme le

'Steve McQueen, Ashes, du 9 janvier 2016 au 27 février 2016, Galerie Marian-Goodman, Paris. 
massacre le plus important depuis la guerre civile, selon le rapport de la commission d'enquête officielle ${ }^{2}$.

Dans un immeuble à deux blocs du Brooklyn Museum, un appartement surchauffé ; un intérieur plein de livres et de papiers, avec sur les murs un ensemble de portraits photographiques où je reconnais mon hôtesse, Elisabeth Fink, aux côtés de personnes dont j'ignore alors majoritairement l'identité - ce sont, je l'apprendrai plus tard, les visages de nombreux militants qui ont marqué les luttes afro-américaines, natives américaines mais aussi ouvrières des années 1970 à celles de 1990 ; il y a aussi des images de célèbres jazzmen elles sont l'œuvre de Larry Fink, célèbre photographe qui est le frère d'Elisabeth -, ainsi que des coupures de presse jaunies sous leur encadrement de fortune : il y est question de procès, de condamnations et de libérations. Elisabeth Fink est une avocate retraitée depuis peu; elle me reçoit en m'expliquant qu'elle se définit elle-même comme une « red jewish brooklyn woman »; elle a été membre de la cellule de Brooklyn du Parti communiste état-uniens, elle a consacré sa vie à la défense des militants accusés par le pouvoir américain de l'avoir menacé ; ses parents étaient eux-mêmes des avocats engagés ; elle a longtemps travaillé aux côtés de William Kunstler, le plus célèbre de ces avocats " gauchistes », présents dans les principaux procès contre les organisations politiques des minorités ${ }^{3}$.

Je suis venu à New York rencontrer Elisabeth Fink, car elle a été la principale avocate des Attica Brothers. Pendant plus de trente ans, l'avocate a mené le combat juridique pour la reconnaissance de la responsabilité de l'État de New York dans le massacre de 29 prisonniers qui avaient pris part à la révolte. Elle a obtenu gain de cause, il y a peu, avec la levée du secret sur un rapport à charge sur les violences commises contre les mutins. Elisabeth parle vite, elle livre mille informations sur cet événement qui est devenu le fil rouge de sa vie. Soudain, tandis que nous parlons des circonstances de l'assaut et de la manière dont il a eu lieu, Elisabeth m'invite à aller dans le couloir d'entrée, à ouvrir le dernier tiroir et à rapporter un énorme dossier de photographies. Nous déplions ce dossier sur la table de sa salle à manger : une cinquantaine de photographies noir et blanc de grand format, 20 par $27 \mathrm{~cm}$ environ. Un premier ensemble de clichés est composé de photographies de la cour au moment de l'assaut, lorsqu'un hélicoptère survole la cour et commence à diffuser un gaz lacrimogène. On y voit les mutins, en plan large, formant foule dans cette cour de terre avec des tranchées et parsemée de petits abris, mais aussi d'autres casqués et armés de simples batons derrière une

\footnotetext{
2 Commission McKay, The Official Report of The New York State Special Commission on Attica, New York, Bantam Book 1972. Sur ces événements, voir : Heather Ann Thompson, Blood in the Water: The Attica Prison Uprising of 1971 and Its Legacy, Pantheon, 2016. ${ }^{3}$ William M. Kunstler, My Life as a Radical Lawyer, New York, Birch Lane Press, 1994.
} 
barricade sur la coursive appelée Times Square. Au fur et à mesure, on distingue de moins en moins leurs silhouettes, la fumée du gaz altérant la netteté. Le reste des photographies a été pris après que les mutins ont été capturés par la Garde nationale, nus et formant une file (the naked snake). L'image a été reprise ensuite en couverture du rapport indépendant qui fut publié en $1972^{4}$. Viennent ensuite deux séries de photographies en noir et blanc. L'une montre le cadavre d'une dizaine de mutins, ceux que l'on voyait vivants sur Times Square. Je songe, en les voyant, à des photographies de victimes de crimes que j'ai beaucoup dépouillées lors de recherches antérieures : chacune des dépouilles est saisie sur le lieu précis de sa mort, une coursive, la cour ; mais sur tous les clichés, ce qui frappe, c'est la présence systématique d'une arme blanche à proximité du corps. La dernière série de clichés, eux aussi en noir et blanc, a été prise dans une morgue : les corps photographiés sont entièrement nus ; ils sont tous meurtris par des blessures par balle et une ardoise avec un numéro a été placée sur le chariot, avant la prise de vue.

Elisabeth qui connaît parfaitement ces photographies n'éprouve pas l'émotion qui me submerge en regardant ces images. J'ai beau avoir vu le film de Cinda Firestone ${ }^{5}$, lu les magazines de l'époque (Newsweek, Time Magazine ...) et de nombreux témoignages, je ne m'attendais pas, cet après-midi de janvier 2015, à me retrouver face à ces images de massacre. Cette émotion est comparable à celle que j'ai ressentie lorsque j'ai vu pour la première fois les photographies d'André A. E. Disderi des communards assassinés pendant la Semaine sanglante en 1871. (photo cadavre dans cercueils ouverts)

Mais cet après-midi, nous sommes seuls avec ces images; elles n'ont jamais été rendues publiques ; je retourne ces photographies cherchant une légende ; en guise d'explication, je ne découvre que le tampon «Confidential», ainsi que pour beaucoup la mention « Grand Jury ». Elisabeth me raconte que ces clichés sont des pièces du procès qui a été intenté à certains mutins par l'État de New York pour leur implication dans la mutinerie qui a coûté la vie à 29 prisonniers et à 10 personnels pénitentiaires. Je comprends très vite que ces clichés ne devraient pas être en possession d'Elisabeth. L'avocate me raconte qu'elle les a volées aux Archives de l'État, aux «Pigs » comme elle dit, reprenant par ce terme le vocabulaire de l'extrême gauche américaine des années 1960-19706. Ces photographies des morts d'Attica ne devaient pas rester dans les mains des bourreaux ; c'était une évidence. Ma certitude sur les archives vascille lorsqu'Elisabeth commente chacune des photographies nommant la victime

\footnotetext{
‘ Attica, the Official Report of the New York State Special Commission on Attica, New York, Paperback, 1972. Cinda Firestone, Attica, États-Unis, 1974, 1h20 min.

Jerry Rubin, Do it !, New York, Simon and Schuster, 1970.
} 
par son prénom, m'expliquant que la série des cadavres armés est fasifiée : les autorités ont ajouté des armes pour justifier le fait d'avoir ouvert le feu sur les mutins.

Elisabeth me propose de prendre cet ensemble pour le montrer en France - avec le centre d'art et éditeur Le Point du jour, nous avons le projet d'une exposition et d'un livre ${ }^{7}$. Je ne m'attendais pas à une telle proposition, moins encore à la disparition d'Elisabeth quelques mois plus tard, me laissant en héritage les photographies des jeunes mutins morts d'Attica.

Que faire de ces clichés ? J'ai passé la frontière avec ces images dans mon sac à dos ; je leur ai fait traversé l'Atlantique. Je suis à présent en charge de ces documents.

Jeune chercheur, lorsque je trouvai une image dans un fond d'archives, il me fallait m'en imprégner pour en garder une image mentale, car le prendre en photo n'était pas autorisé et je savais que la seule description écrite ne suffirait pas ; si besoin, je pouvais commander un tirage aux services de reproduction, plus tard et à grand frais. Cette séquence apparition/disparition/réapparition, je l'ai vécue au tout début des années 1990 dans le fond Alexandre Lacassagne à la Bibliothèque municipale de Lyon. J'y travaillais sur des autobiographies de criminels suscitées; après avoir lu leurs récits de vies, cherchant à documenter les affaires judiciaires qui les avaient menés en prison, je me souviens avoir découvert les portraits de deux d'entre eux - ceux d'Henri Vidal et d'Émile Nouguier. Je les trouvai dans des publications contemporaines, des monographies sur les apaches et les sadiques. Ces images étaient en série, déformées, comme écrasées par le discours...

Il en alla autrement du plus anonyme, Charles Double, qui avait tué sa mère et s'était ensuite enfui avec son amant jusqu'en Égypte ; il était l'auteur d'un texte singulier « État mental d'un inverti parricide »; au détour de son manuscrit autobiographique, je me suis retrouvé face à face avec deux portraits photographiques de Charles Double, dont le cliché a sans doute été pris dans l'atelier d'un photographe : Double y prend la pose, il est élégamment vêtu, porte une lavallière... Ces deux images disparaissent derrière ses cahiers, je suis pris par la transcription de ses cahiers, comme si les sources de l'historien ne pouvaient être que textuelles, comme si l'image était une source secondaire, voire anecdotique. J'oublie ces clichés, d'autant que sur le microfilm auquel j'ai accès ensuite, la photographie a été reproduite en positif et par conséquent lorsque je l'imprime, son portrait est invisible. Rétrospectivement, cette disparition me semble pleine de sens. Je ne voulais pas échanger de

Philippe Artières, dir., ATTICA USA 1971, textes de Nicole Brenez, Thierry Gervais, Tom Holt, Emmanuel Parent, Caroline Rolland-Diamond, Jedediah Sklower et Elvan Zabunyan, Cherbourg/Paris, Le Point du jour, 2017. 
regards avec Charles Double, non pas seulement parce que j'avais transgressé l'interdit qu'il avait soigneusement pris la peine d'inscrire sur la couverture de ses cahiers : «Pour être lu par des docteurs en médecine », mais sans doute parce que cette photographie me mettait face à mon impuissance ; j'étais formé à lire des textes, et non à regarder et à analyser des images. Sur la première édition du texte de Double (aux éditions du Gay Kitsch Camp, dirigées par Patrick Cardon), j'ai ainsi reproduit «bêtement» un portrait de l'inverti parricide en médaillon, avec une fonction illustrative. Ce n'est que plus tard, lorsque j'ai commencé à appréhender ces photographies comme participant non pas seulement à une histoire du crime, mais à une histoire des images que je me suis mis à regarder Charles Double, mesurant combien en cette fin de $\mathrm{XIX}^{\mathrm{e}}$ siècle l'écriture manuscrite et le portrait photographique étaient en concurrence dans leur rapport à la vérité, combien ces archives croisaient l'histoire des images - aussi, ai-je choisi, quelques années plus tard, de placer en couverture du Livre des vies coupables ${ }^{8}$ le portrait « iconique » du condamné Lewis Peyne, prise en 1865 et reproduite par Roland Barthes dans son essai La Chambre claire.

Lorsque vingt ans plus tard, dans les archives judiciaires de Rome, enquêtant sur un assassin, un dénommé Bambino Marchi, ayant assassiné un philosophe jésuite dans une rue de Rome un jour de l'automne 1925, je trouvai dans son expertise mentale une photographie de lui, j'étais soucieux de cette dimension. Mais elle m'interloqua. C'était une bien «étrange» image - «étrange » car la scène saisie était énigmatique : Bambino Marchi posait, cigarette aux lèvres, devant ce qui semblait être un mannequin pendu par la gorge avec la corde d'un puits; étrange aussi était le document, car rien ne permettait de savoir dans quelle circonstance cette photographie avait été prise, ni par qui. S'agissait-il d'une reconstitution d'un précédent meurtre? D'une mise en scène ludique comme celle que j'avais rencontrée en travaillant sur les portraits de Thérèse de Lisieux où la carmélite s'était fait photographier déguisée en Jeanne d'Arc ? La photographie numérique s'étant développée, l'interdiction de photographier les documents avait été levée; je me souviens d'avoir pris grand soin pour photographier cette image. Dans le récit que je rédigeai ensuite, ce portrait joua un rôle clé, et marqua le moment de basculement du récit du passé au présent. Dans le livre que je publiai, il est l'unique image reproduite ${ }^{9}$. Tout se passe comme si cette photographie résistait par son inconguité, son caratère extraordinairement insolite, à toute interprétation - comme si là, dans ce document, se tenait aussi une opacité sur laquelle l'historien butait, et qui n'était pourtant

' Philippe Artières, Le Livre des vies coupables. Autobiographies de criminels (1896-1909), Paris, Éditions Albin Michel, 2000.

' Philippe Artières, Vie et mort de Paul Geny, Paris, Éditions du Seuil, coll. « Fictions et Cie », 2013. 
pas hors de l'Histoire, mais bien en son centre. La photographie de Bambino y faisait événement.

Aussi lorsque nous avons entrepris avec Mathieu Pernot d'explorer les archives de l'hôpital psychiatrique, la Fondation du Bon-sauveur de Picauville dans la Manche, cette question fut centrale ${ }^{10}$. Notre association - un photographe et un historien - obligeait à prendre au sérieux cette question de la photographie comme archives : comment une histoire des images croisaitelle tout à la fois l'histoire de la psychiatrie, celle de la Seconde Guerre mondiale, celle des loisirs... ? La photographie n'était plus source, elle devenait objet d'histoire ; une histoire de l'infra-ordinaire des images dans l'institution extraordinaire que constitue l'hôpital psychiatrique tenu par une communauté de religieuse. Sauf à nous livrer à un récit anecdotique, il nous fallait faire l'archéologie de ces images, comprendre les différentes circonstances de leur production (fêtes rituelles, kermesses, sorties, vacances...), mais aussi les modes et lieux de leur diffusion (projections, tirages...): un autre visage de cette communauté composée de psychiatrisés et de religieuses y apparaissait. Une histoire sensible qui faisait place à des figures anonymes de femmes et d'hommes anonymes.

Parmi eux, il y a ce jeune homme endormi sur la banquette de ce qui semble être un bateau. Dort-il ou songe t-il au juste ? C'est un jour de sortie, peut-être une journée de visite des îles anglo-normandes, non loin de la dépendance de l'hôpital où les patients viennent séjourner chaque été. Impossible de mettre un nom ou un prénom sur ce visage et de lui assigner un des nombreux dossiers médicaux que j'ai pu consulter. Le jeune homme n'apparait pas ailleurs, nous n'avons que ce portrait photographique de lui.

Nous décidons de faire une exception et de réaliser un tirage papier de cette diapositive couleur et de l'exposer en ouverture de la première salle. Cet énorme corpus de plusieurs milliers de photographies réalisé au sein de l'institution du Bon-Sauveur a le même statut que le portrait du jeune homme : au premier regard d'une extraordinaire banalité, mais pour qui veut bien le voir, marqué par un trouble très léger, presque invisible. La photographie se fait écriture de l'histoire.

Elles marchent sur une route ; un groupe de femmes avance, déterminées, revêtues de chauds manteaux; ce doit être le début des années 1960; elles semblent manifester; on lit difficilement sur un tract qu'elles brandissent : «Moins de milliards pour la force de frappe, des sous pour les mineurs. » Le cliché noir et blanc, de format 6/6, appartient à une série

${ }^{10}$ Philippe Artières, L'Asile des photographies, avec Mathieu Pernot, Cherbourg, Le Point du jour, 2013. 
d'images des manifestations de femmes de mineurs en soutien à leurs maris en grève en 1963. Cette image rappelle ce que le récit historique a laissé sur le côté du chemin, l'importance des femmes dans la grande grève des houillères de Lorraine et du Nord lors de cette longue grève $^{11}$. Insatisfaites des réponses fournies par le Préfet de Moselle, ces femmes décident d'aller voir le Général de Gaulle. Le 27 mars 1963, elles télégraphient à la Présidence de la République leurs venues le lendemain; le 28, les épouses et mères quittent Merlebach à 5 heures du matin; arrivées aux portes de Paris à 13 heures, un déjeuner leur est offert dans une école municipale de Pantin; elles rejoignent en bus le $8^{\mathrm{e}}$ arrondissement, sous les acclamations de certains automobilistes ; mais, à quelques dizaines de mètres de l'Élysée, trois femmes de mineurs sont autorisées à descendre. Elles ne sont pas reçues par Charles de Gaulle, tout juste par le portier du Palais. Les manifestantes ne désarment pas et tentent alors la même démarche auprès du Premier ministre, mais c'est aussi le portier qui les reçoit à Matignon. Le lendemain, de retour dans le bassin houiller, elles disent leur colère devant ce « mépris » et poursuivent leur mobilisation. Quelques semaines plus tard, les revendications des mineurs sont acceptées, mais le Général de Gaulle de déclarer : «Des grèves commes celles-ci ne peuvent recommencer avant plusieurs années. Et dans plusieurs années, l'heure du charbon sera passée. » La photographie de ces femmes en Lorraine, prise par un correspondant de l'Humanité, ne fut jamais publiée. Elle est conservée aux Archives de SeineSaint-Denis, à Bobigny. Ce cliché est mémoire d'une histoire tue. Il en est de même des dizaines de photographies prises par le photographe du Républicain-Lorrain de la mutinerie à la prison de Charles III de Nancy le 15 janvier 1972. Ses clichés font traces : montés sur le toit de l'établissement pénitentiaire, en plein centre-ville, les mutins s'étaient pour la première fois rendus visibles: ils avaient dialogué avec la foule au dehors, ils avaient brandi une banderole, sur laquelle on pouvait lire les simples mots : «On a faim. » Le photographie avait figé cet instant du soulèvement ${ }^{12}$. Elle en est l'unique archive, comme du soulèvement des femmes de mineurs.

C'est nourri de ces multiples expériences de regard - celles sur le portrait de Charles Double, de l'image de Bambino Marchi, sur le cliché du jeune homme endormi et sur la photographie des femmes de mineurs -, que j'ai appréhendé les images des mutins morts d'Attica. Dans l'exposition Attica, USA, 1971, comme dans le livre éponyme, ces photos sont montrées collectivement comme fut la mutinerie, sans encadrement pour ne pas neutraliser leur statut

\footnotetext{
"Philippe Artières, Au fond, Paris, Éditions du Seuil, Coll. «Fictions et Cie », 2016.

"La Révolte de la prison de Nancy. 15 janvier 1972. Photographies de Gérard Drolc, Elie Kagan, Martine Franck, peintures de Gérard Fromanger..., Paris/Cherbourg, Le Point du jour éditeur, 2013.
} 
d'archives et enfin sans éclairage afin, non seulement de les distinguer des photographies d'auteurs (dont celles de Danny Lyons ou de Cornell Capa), mais surtout de tenter d'ériger pour ces mutins un tombeau qui ne soit pas monument mais soulèvement. Manier ainsi les images, c'est tenter de répondre à l'injonction faite à l'historien.ne de prendre soin des morts, et plus encore des morts exclus du récit historique, dont la mémoire n'est transmise qu'oralement : ici, en 1975, dans un film de Sydney Lumet, par la bouche d'Al Pacino' ${ }^{13}$, ailleurs par celle de John Travolta dans La Fièvre du samedi soir en 1977.

"sydney Lumet, Dog Day Afternoon, États-Unis, 1975, 2h30 min. 